Pacific Journal of Mathematics

ON A CLASS OF MEROMORPHIC FUNCTIONS WITH 


\section{ON A CLASS OF MEROMORPHIC FUNCTIONS WITH DEFICIENT ZEROS AND POLES}

\section{S. HeLlerstein}

Introduction. It has been shown by A. Edrei and W.H.J. Fuchs [1], that if $f$ is an entire function all of whose zeros lie on the negative real axis, then $f$ has zero as a Nevanlinna deficient value provided only that the exponent of convergence of the zeros is finite and greater than 1. The extension of this result to more general distributions of the arguments of the zeros and poles of a meromorphic function was investigated independently in [2] and [3].

In [2], Edrei, Fuchs and the present author consider entire functions whose zeros have a finite exponent of convergence and are distributed on a finite number of rays. The main result of that investigation is the following:

Theorem A. Let $f(z)$ be entire. Assume that all its zeros $\left\{a_{\mu}\right\}$ lie on the radii defined by

$$
r e^{i \omega_{0}}, r e^{i \omega_{1}}, \cdots, r e^{i \omega_{n}} \quad(r>0)
$$

where the $\omega$ 's are real.

Then there exists a positive constant $K$, depending only on the $\omega$ 's and such that the condition

$$
\sum_{\mu} \frac{1}{\left|a_{\mu}\right|^{K}}=+\infty
$$

and the condition

$$
\sum_{\mu} \frac{1}{\left|a_{\mu}\right|^{\xi}}<+\infty
$$

for some finite value of $\xi$, imply

$$
\delta(0, f) \geqq A>0
$$

where $A(>0)$ is an absolute constant.

In [3], A. A. Goldberg shows that given $\rho$ not an integer, such that $\frac{1}{2}<\rho<+\infty$ and given two arbitrary positive numbers $\alpha$ and $\beta$

Received June 15, 1962. This constitutes the first chapter of the author's dissertation entitled "Deficiencies of a Meromorphic Function and Distribution of Values in Angles and Annuli"; Syracuse University, 1961. The author wishes to thank Professor A. Edrei for his guidance.

This work was supported in part by the United States Air Force, Contract No. AF 49 (638)-571, Monitored by the Office of Scientific Research. 
then there exists a meromorphic function $f(z)$ of order $\rho$, all of whose zeros lie on the rays $\arg z=\alpha$ and $\arg z=\alpha+(\pi / \rho)$ and all of whose poles lie on the rays $\arg z=\beta$ and $\arg z=\beta+(\pi / \rho)$ for which $\delta(0, f)=$ $\delta(\infty, f)=0$.

Hence, any theorem for meromorphic functions analogous to Theorem A must place some restriction on the geometrical configuration of the rays on which the zeros and poles of the function are situated.

The main purpose of this note is to show that the methods of [2] go further and yield the following generalization of Theorem A to meromorphic functions.

THEOREM. Let $f(z)$ be meromorphic. Let $\left\{a_{\mu}\right\}$ denote the zeros of $f$ which lie on the radii defined by

$$
r e^{i \omega_{1}}, r e^{i \omega_{2}}, \cdots, r e^{i \omega_{m}} \quad(r>0)
$$

and let $\left\{b_{\nu}\right\}$ denote the poles of $f$ which lie on the radii defined by

$$
r e^{i \gamma_{1}}, r e^{i \psi_{2}}, \cdots, r e^{i \psi_{n}} \quad(r>0)
$$

Assume

(iii) that the real numbers $2 \pi, \omega_{1}, \cdots, \omega_{k}, \psi_{1}, \cdots, \psi_{n}$ $(0 \leqq k \leqq m)$ are linearly independent over the field of rational numbers and

$$
\omega_{k+h}=a_{0, h} 2 \pi+\sum_{i=1}^{k} a_{i, h} \omega_{i} \quad(h=1,2, \cdots, m-k)
$$

where $a_{0, h}, a_{1, h}, \cdots, a_{k, h}$ are rational.

Let $\left\{a_{\mu}^{*}\right\}$ denote the zeros of $f$ which do not lie on the radii defined by (i) and $\left\{b_{\nu}^{*}\right\}$ the poles of $f$ which do not lie on the radii defined by (ii).

Then, there exists a positive constant $K$ (depending only on the $\omega$ 's and $\psi$ 's) and an absolute constant $B(>0)$ such that the conditions

$$
\begin{aligned}
& \sum_{\mu} \frac{1}{\left|a_{\mu}\right|^{K}}+\sum_{\nu} \frac{1}{\left|b_{\nu}\right|^{K}}=+\infty \\
& \sum_{\mu} \frac{1}{\left|a_{\mu}\right|^{\xi}}+\sum_{\nu} \frac{1}{\left|b_{\nu}\right|^{\xi}}<+\infty
\end{aligned}
$$

for some finite value of $\xi(>K)$ and

$$
\sum_{\mu} \frac{1}{\left|a_{\mu}^{*}\right|^{\eta}}+\sum_{\nu} \frac{1}{\left|b_{\nu}^{*}\right|^{\eta}}<+\infty
$$

for some $\eta<B$, imply 


$$
\varlimsup_{r \rightarrow+\infty} \frac{N(r, f)+N(r, 1 / f)}{T(r, f)} \leqq \frac{1}{1+A}
$$

where $A(>0)$ is an absolute constant.

As an immediate consequence of this theorem and the definition of deficiency, we find

COROLlaRY. The assumptions of the theorem imply

$$
\delta(0, f) \geqq \frac{A}{1+A} ; \quad \delta(\infty, f) \geqq \frac{A}{1+A} .
$$

We shall show that Lemma 4 of [2] combined with a suitable number theoretical lemma which we state and prove in $\S 3$ are sufficient to yield our theorem.

2. Statement of a known lemma. For the convenience of the reader, we restate Lemma 4 of [2] in a form suitable for use here.

LEMmA A. Let $f(z)$ be a meromorphic functions of genus not greater than 2.

Assume

(i) that its zeros $\left\{a_{\mu}\right\}$ lie in the region defined by

$$
|\arg z| \leqq \frac{\pi}{60}
$$

(ii) that its poles $\left\{b_{\nu}\right\}$ lie in the region defined by

$$
\begin{gathered}
|\arg z-\pi| \leqq \frac{\pi}{60}, \\
\sum_{\mu} \frac{1}{\left|a_{\mu}\right|}+\sum_{\nu} \frac{1}{\left|b_{\nu}\right|}=+\infty .
\end{gathered}
$$

Then, for all sufficiently large values of $r$,

$$
T(r, f) \geqq(1+A)\left\{N\left(r, \frac{1}{f}\right)+N(r, f)\right\}
$$

where $A(>0)$ is an absolute constant.

The inequality (2.1) still holds if $f(z)$ is replaced by $F(z)$ :

$$
F(z)=e^{s(z)} f(z)
$$

where $S(z)$ is an entire function (which may reduce to a polynomial). 
3. A number theoretical lemma. In order to apply the methods of [2], we also require the following generalization of a number theoretical argument used in the proof of Theorem $A[2, \S 6]$.

Conventions. Before we proceed with the statement and proof of the lemma, we make the following conventions.

In all that follows, we shall use the terms "linear dependence" and "linear independence" to denote linear dependence and independence over the field of rational numbers.

In addition, given a set $S$ of real numbers we shall use the term " $S$ * is a maximal linearly independent subset of $S$ " to mean the following:

(i) the elements of $S^{*}$ are linearly independent over the field of rational numbers, and

(ii) any element of $S$ is a linear combination with rational coefficients of the elements of $S^{*}$.

LEMMA 1. Let $S_{1}$ be the set of real numbers

$$
2 \pi, \omega_{1}, \omega_{2}, \cdots, \omega_{m}
$$

and $S_{2}$ the set consisting of the real numbers

$$
\psi_{1}, \psi_{2}, \cdots, \psi_{n}
$$

\section{Assume}

(i) that the set of real numbers $2 \pi, \omega_{1}, \cdots, \omega_{k},(0 \leqq k \leqq m)$ is a maximal linearly independent subset of $S_{1}$,

(ii) that the real numbers

$$
2 \pi, \omega_{1}, \omega_{2}, \cdots, \omega_{k}, \psi_{1}, \psi_{2}, \cdots, \psi_{n}
$$

are linearly independent.

Then

given $\varepsilon(>0)$ there exists an increasing sequence of positive integers $\left\{L_{s}\right\}_{s=1}^{\infty}$ and sequences of positive integers $\left\{M_{s, i}\right\}_{s=1}^{\infty}, \quad(i=1,2, \cdots, m)$; $\left\{N_{s, j}\right\}_{s=1}^{\infty}(j=1,2, \cdots, n)$; such that for $s=1,2,3, \cdots$

$$
\begin{array}{ll}
\left|L_{s} \omega_{i}-2 \pi M_{s, i}\right|<\varepsilon, & (i=1,2, \cdots, m), \\
\left|L_{s} \psi_{j}-\left(2 N_{s, j}+1\right) \pi\right|<\varepsilon, & (j=1,2, \cdots, n),
\end{array}
$$

and all $s \geqq s_{0}$

$$
\frac{L_{s+1}}{L_{s}} \leqq 2
$$


Proof. We assume $k<m$ and prove the lemma for this case only. If $k=m$, it will be clear that one part of our argument yields the desired result.

By assumption (i), $\omega_{k+h}(h=1,2, \cdots, m-k)$ is a linear combination with rational coefficients of $2 \pi, \omega_{1}, \omega_{2}, \cdots, \omega_{k}$. Hence, there exist a positive integer $T$ and integers $A_{h, i},(h=1,2, \cdots, m-k),(i=0,1, \cdots, k)$, such that

$$
T \omega_{k+h}=2 \pi A_{h, 0}+\sum_{i=1}^{k} A_{h, i} \omega_{i} \quad(h=1,2, \cdots, m-k) .
$$

Set

$$
\begin{gathered}
\bar{A}_{h}=\sum_{i=1}^{k}\left|A_{h, i}\right| \quad(h=1,2, \cdots, m-k), \\
Q=\max \left\{T, \bar{A}_{1}, \bar{A}_{2}, \cdots, \bar{A}_{m-k}\right\}
\end{gathered}
$$

and

$$
T=2^{I}(2 J+1)
$$

where $I$ and $J$ are nonnegative integers.

Assumption (ii) of our lemma and the equidistribution theorem of $\mathrm{H}$. Weyl [4] imply that there exists a positive increasing sequence of integers $\left\{B_{s}\right\}_{s=1}^{\infty}$, and sequences of integers $\left\{C_{s, i}\right\}_{s=1}^{\infty},(i=1,2, \cdots, k)$, and $\left\{D_{s, j}\right\}_{s=1}^{\infty},(j=1,2, \cdots, n)$, such that for each $s=1,2, \cdots$

$$
\begin{array}{cc}
\left|B_{s} \omega_{i}-2 \pi C_{s, i}\right|<\frac{\varepsilon}{Q} & (i=1,2, \cdots, k), \\
\left|B_{s} \psi_{j}-2 \pi D_{s, j}-\frac{\pi}{2^{I}}\right|<\frac{\varepsilon}{T} & (j=1,2, \cdots, n),
\end{array}
$$

and $\left\{B_{s}\right\}_{s=1}^{\infty}$ has a positive density; that is

$$
\lim _{s \rightarrow+\infty} \frac{s}{B_{s}}=d>0 \text {. }
$$

From (3.8) and (3.7) we have for each $s=1,2,3, \cdots$

$$
\left|B_{s} T \omega_{i}-2 \pi T C_{s, i}\right|<\frac{\varepsilon T}{Q} \leqq \varepsilon .
$$

From (3.4), (3.8), (3.5), and (3.6), we deduce that for $s=1,2,3, \cdots$

$$
\begin{aligned}
& \left|B_{s} T \omega_{k+h}-2 \pi B_{s} A_{h, 0}-2 \pi \sum_{i=1}^{k} A_{h, i} C_{s, i}\right| \\
& \quad \leqq\left[\max _{i=1,2, \cdots, k}\left\{\left|B_{s} \omega_{i}-2 \pi C_{s, i}\right|\right\}\right] \cdot \sum_{i=1}^{k}\left|A_{h, i}\right| \\
& \quad<\frac{\varepsilon}{Q} \cdot Q=\varepsilon \quad(h=1,2,3, \cdots, m-k) .
\end{aligned}
$$


Furthermore, (3.7) and (3.9) imply that for each $s=1,2,3, \cdots$

$$
\mid B_{s} T \psi_{j}-2 \pi T D_{s, j}-\pi(2 J+1)_{1}<\varepsilon .
$$

We now show that for $s \geqq s_{0}$,

$$
\frac{B_{s+1}}{B_{s}} \leqq 2 \text {. }
$$

If this were not so, we would have for infinitely many values of $s$ :

$$
\begin{aligned}
& \frac{1}{2} \cdot \frac{1}{B_{s}}>\frac{1}{B_{s+1}}, \\
& \frac{1}{2} \cdot \frac{s}{B_{s}}>\frac{s+1}{B_{s+1}} \cdot \frac{s}{s+1},
\end{aligned}
$$

and by (3.10)

$$
\frac{1}{2} d \geqq d,
$$

which is impossible.

We set $L_{s}=B_{s} T$. The inequalities (3.11), (3.12), (3.13) and (3.14) show that the sequence $\left\{L_{s}\right\}_{s=1}^{\infty}$ satisfies all the assertions of our lemma (with an obvious choice of the corresponding sequences $\left\{M_{s, i}\right\}$, and $\left\{N_{s, j}\right\}$ ).

4. Proof of the theorem. From the hypotheses of our theorem, it follows that if we set $\varepsilon=\pi / 60$ in Lemma 1 , there exist sequences $\left\{L_{s}\right\}_{s=1}^{\infty},\left\{M_{s, i}\right\}_{s=1}^{\infty},(i=1,2, \cdots, m),\left\{N_{s, j}\right\}_{s=1}^{\infty},(j=1,2, \cdots, n)$ such that (3.1), (3.2) and (3.3) hold with $\varepsilon=\pi / 60$.

Now choose $K=L_{s_{0}}$ and note that $L_{s_{0}}$ depends only on the $\omega$ 's and $\psi$ 's. Since the zeros $\left\{a_{\mu}\right\}$ and poles $\left\{b_{\nu}\right\}$ of $f$ satisfy conditions (1.1) and (1.2), it follows that there exists an integer $q \geqq K$ such that

$$
\sum_{\mu} \frac{1}{\left|a_{\mu}\right|^{q}}+\sum_{\nu} \frac{1}{\left|b_{\nu}\right|^{q}}=+\infty
$$

and

$$
\sum_{\mu} \frac{1}{\left|a_{\mu}\right|^{q+1}}+\sum_{\nu} \frac{1}{\left|b_{\nu}\right|^{q+1}}<+\infty .
$$

Define $h$ by the inequalities

$$
L_{h} \leqq q<L_{h+1} .
$$

Clearly

$$
h \geqq K=L_{s_{0}},
$$

and in view of (3.3) 


$$
q<2 L_{h} .
$$

Consider the auxiliary function

$$
\hat{f}(z)=e^{S(z)} \frac{\pi_{1}(z)}{\pi_{2}(z)}
$$

where $S(z)$ is an arbitrary entire function,

$$
\pi_{1}(z)=\prod_{\mu}\left(1-\frac{z}{a_{\mu}}\right) \exp \left(\frac{z}{a_{\mu}}+\frac{z^{2}}{2 a_{\mu}^{2}}+\cdots+\frac{z^{q}}{q a_{\mu}^{q}}\right)
$$

and

$$
\pi_{2}(z)=\prod_{\nu}\left(1-\frac{z}{b_{\nu}}\right) \exp \left(\frac{z}{b_{\nu}}+\frac{z^{2}}{2 b_{\nu}^{2}}+\cdots+\frac{z^{q}}{q b_{\nu}^{q}}\right) ;
$$

where in view of (4.1) and (4.2), at least one of the two products (4.5) and (4.6) is canonical.

We show next that (4.4) holds for a function of the form $\hat{f}$, and finally that this implies the validity of (4.4) for $f$.

Put $L_{h}=L$ and consider the function

$$
F(z)=\hat{f}(z) \hat{f}(\omega z) \cdots \hat{f}\left(\omega^{L-1} z\right)
$$

where $\omega=e^{(2 \pi i) / L}$.

It is an easy consequence of the relations (4.4)-(4.7) that

$$
F(z)=G\left(z^{L}\right)=e^{R\left(z^{L}\right)} g\left(z^{L}\right)=e^{R\left(z^{L}\right)} \frac{\prod_{\mu}\left(1-\frac{z}{a_{\mu}^{L}}\right) \exp \left(\frac{z^{L}}{a_{\mu}^{L}}\right)}{\prod_{\nu}\left(1-\frac{z^{L}}{b_{\nu}^{L}}\right) \exp \left(\frac{z^{L}}{b_{\nu}^{L}}\right)}
$$

where $R$ is entire and $g$ is a meromorphic function of genus not greater than one. In fact, our assumptions imply that the genus of $g$ is actually one. In order to see this we observe first that for all $\mu$ and $\nu$

$$
a_{\mu}^{L} \neq b_{\nu}^{L} .
$$

For, if this were not so, we would have for some $\mu$ and $\nu$

$$
\begin{aligned}
a_{\mu}^{L} & =b_{\nu}^{L}, \\
\left|a_{\mu}\right|^{L} e^{i L \omega_{j}} & =\left|b_{\nu}\right|^{L} e^{i L \psi_{k}} .
\end{aligned}
$$

Hence, for some integer $N$

$$
L \psi_{k}=L \omega_{j}+2 N \pi,
$$

which, in view of conditions (iii) and (iv) of the theorem, is impossible. 
Therefore, cancellation of zeros and poles of $g$ cannot occur. It follows then from (4.1), (4.2) and the inequality $L \leqq q<2 L$, that the genus of $g$ is indeed one.

Setting $z^{L}=\zeta$

$$
G(\zeta)=e^{R(\zeta)} \frac{\prod_{\mu}\left(1-\frac{\zeta}{a_{\mu}^{L}}\right) \exp \left(\frac{\zeta}{a_{\mu}^{L}}\right)}{\prod_{\nu}\left(1-\frac{\zeta}{b_{\nu}^{L}}\right) \exp \left(\frac{\zeta}{b_{\nu}^{L}}\right)} .
$$

But $L=L_{h}$ was so chosen that there exist positive integers $M_{i}$, $(i=1,2, \cdots, m)$ and $N_{j},(j=1,2, \cdots, n)$ so that

$$
\left|L \omega_{i}-2 \pi M_{i}\right|<\frac{\pi}{60} \quad(i=1,2, \cdots, m)
$$

and

$$
\left|L \psi_{j}-\left(2 N_{j}+1\right) \pi\right|<\frac{\pi}{60} \quad(j=1,2, \cdots, n) .
$$

Hence $G(\zeta)$ is a meromorphic function satisfying the hypotheses of Lemma $\mathrm{A}$, and consequently

$$
T(r, G(\zeta)) \geqq(1+A)\left\{N\left(r, \frac{1}{G(\zeta)}\right)+N(r, G(\zeta))\right\} \quad\left(r \geqq r_{0}\right) .
$$

We observe now, that the fundamental definitions of the theory imply [2, p. 147] that for any meromorphic function $W(z)$

$$
N\left(r, W\left(z^{L}\right)\right)=N\left(r^{L}, W(z)\right)
$$

and

$$
T\left(r, W\left(z^{L}\right)\right)=T\left(r^{L}, W(z)\right) .
$$

Since $G(\zeta)=G\left(z^{L}\right)=F(z)$, we deduce from (4.12), (4.13) and (4.14) that

$$
T(r, F(z)) \geqq(1+A)\left\{N\left(r, \frac{1}{F(z)}\right)+N(r, F(z))\right\} \quad\left(r \geqq r_{0}\right) .
$$

Now, the definition of $\omega$, conditions (i)-(iv) of the hypothesis and the definitions (4.4)-(4.6) prevent the possibility of cancellation between the zeros of one of the functions $\hat{f}\left(\omega^{j} z\right)(j=0,1,2, \cdots, L-1)$ and the poles of another of these functions.

Hence, by (4.7) and the basic definitions of Nevanlinna's theory, it follows that

$$
N(r, F(z))=L N(r, \hat{f}(z))
$$




$$
N\left(r, \frac{1}{F(z)}\right)=L N\left(r, \frac{1}{\hat{f}(z)}\right)
$$

and

$$
T(r, F(z))=L T(r, \hat{f}(z)) .
$$

From (4.15), (4.16), (4.17) and (4.18) we readily deduce

$$
T(r, \hat{f}(z)) \geqq(1+A)\left\{N\left(r, \frac{1}{\hat{f}(z)}\right)+N(r, \hat{f}(z))\right\} .
$$

The inequality (4.19) together with the definition of deficiency imply

$$
\delta(0, \widehat{f}) \geqq \frac{A}{1+A}, \quad \delta(\infty, \hat{f}) \geqq \frac{A}{1+A} .
$$

Hence, by Theorem 4 of [1], it follows that the lower order $\lambda$ of $f(z)$ satisfies

$$
\lambda \geqq \frac{\log \left[1+\frac{A^{2}}{1+2 A}\right]}{\log \left[1+\frac{4(1+A)^{2}}{A}\right]}=B>0 .
$$

Since $A(>0)$ is an absolute constant, the same is true of $B$.

We now return to $f(z)$. Assume that (1.3) holds for some $\eta<B$; $B$ defined by (4.21).

Then, in view of the assumptions of the theorem, we may represent $f(z)$ in the form

$$
f(z)=h(z) \hat{f}_{0}(z)
$$

where $h(z)$ is a meromorphic function of order less than $B$ and $\hat{f}_{0}(z)$ is defined by (4.4) with a suitable choice of $S(z),(4.5)$ and (4.6). Hence it follows that (4.19) holds with $f$ replaced by $\hat{f}_{0}$; moreover, the lower order of $\hat{f}_{0}$ exceeds the order of $h$.

Then, by elementary inequalities of Nevanlinna's theory

$$
\begin{gathered}
T\left(r, h \hat{f}_{0}\right) \sim T\left(r, \hat{f}_{0}\right), \\
\frac{N\left(r, h \hat{f}_{0}\right)}{T\left(r, h \hat{f}_{0}\right)}=\frac{N\left(r, \hat{f}_{0}\right)}{T\left(r, \hat{f}_{0}\right)}+o(1)
\end{gathered}
$$

and

$$
\frac{N\left(r, \frac{1}{h \hat{f}_{0}}\right)}{T\left(r, \hat{f}_{0}\right)}=\frac{N\left(r, \frac{1}{\hat{f}_{0}}\right)}{T\left(r, \hat{f}_{0}\right)}+o(1) .
$$


The inequality (1.4) is now an immediate consequence of (4.19) applied to $\hat{f}_{0}(z)$, together with the relations (4.22), (4.23) and (4.24).

\section{REFERENCES}

1. A Edrei and W. H. J. Fuchs, On the growth of meromorphic functions with several deficient values, Trans. Amer. Math. Soc., 93 (1959), 292-328.

2. A. Edrei, W. H. J. Fuchs and S. Hellerstein, Radial distribution and deficiencies of the values of a meromorphic function, Pacific J. Math., 2 (1961), 135-151.

3. A. A. Goldberg, Distribution of Values of meromorphic functions with separated zeros and poles, Soviet Mathematics, Vol. 2, No. 2 (1961), 389-392.

4. H. Weyl, Über die Gleichverteilung von Zahlen mod. Eins, Math. Annalen 77 (1916), 313-352. 


\section{PACIFIC JOURNAL OF MATHEMATICS}

\section{EDITORS}

\section{RalPh S. Phillips}

Stanford University

Stanford, California

M. G. Arsove

University of Washington

Seattle 5, Washington
J. Dugundji

University of Southern California Los Angeles 7, California

Lowell J. Paige

University of California

Los Angeles 24, California

\section{ASSOCIATE EDITORS}
E. F. BECKENBACH
D. DERRY
H. L. ROYDEN
E. G. STRAUS
T. M. CHERRY
M. OHTSUKA
E. SPANIER
F. WOLF

\section{SUPPORTING INSTITUTIONS}

\author{
UNIVERSITY OF BRITISH COLUMBIA \\ CALIFORNIA INSTITUTE OF TECHNOLOGY \\ UNIVERSITY OF CALIFORNIA \\ MONTANA STATE UNIVERSITY \\ UNIVERSITY OF NEVADA \\ NEW MEXICO STATE UNIVERSITY \\ OREGON STATE UNIVERSITY \\ UNIVERSITY OF OREGON \\ OSAKA UNIVERSITY \\ UNIVERSITY OF SOUTHERN CALIFORNIA
}

STANFORD UNIVERSITY

UNIVERSITY OF TOKYO

UNIVERSITY OF UTAH

WASHINGTON STATE UNIVERSITY

UNIVERSITY OF WASHINGTON

AMERICAN MATHEMATICAL SOCIETY

CALIFORNIA RESEARCH CORPORATION SPACE TECHNOLOGY LABORATORIES

NAVAL ORDNANCE TEST STATION

Printed in Japan by International Academic Printing Co., Ltd., Tokyo Japan 


\section{Pacific Journal of Mathematics}

\section{Vol. 13, No. 1 \\ March, 1963}

Frantz Woodrow Ashley, Jr., A cone of super-(L) functions............. 1

Earl Robert Berkson, Some metrics on the subspaces of a Banach space....

Felix Earl Browder and Walter Strauss, Scattering for non-linear wave

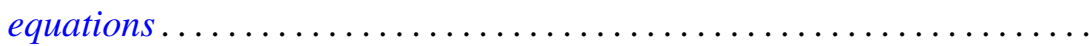

Edmond Darrell Cashwell and C. J. Everett, Formal power series ..........

Frank Sydney Cater, Continuous linear functionals on certain topological

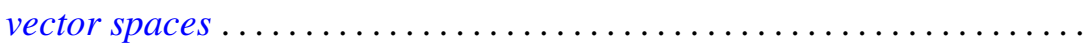

John Douglas Dixon, General group extensions ....................

Robert Pertsch Gilbert, On harmonic functions of four variables with

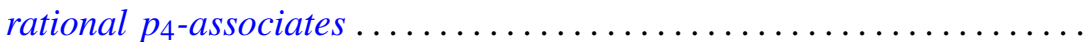

Irving Leonard Glicksberg, On convex hulls of translates ..............

Simon Hellerstein, On a class of meromorphic functions with deficient zeros

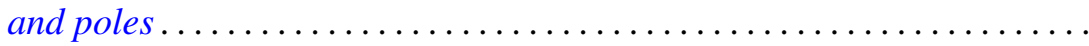

Donald William Kahn, Secondary cohomology operations which extend the

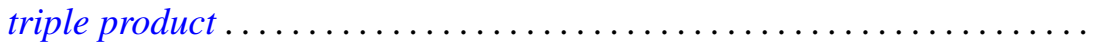

G. K. Leaf, A spectral theory for a class of linear operators .............

R. Sherman Lehman, Algebraic properties of the composition of solutions of partial differential equations ........................... 157

Joseph Lehner, On the generation of discontinuous groups ............. 169

S. P. Lloyd, On certain projections in spaces of continuous functions ...... 171 Fumi-Yuki Maeda, Generalized spectral operators on locally convex spaces ..................................

Donald Vern Meyer, $E^{3}$ modulo a 3-cell

William H. Mills, An application of linear programming to permutation groups.

Richard Scott Pierce, Centers of purity in abelian groups

Christian Pommerenke, On meromorphic starlike functions ...

Zalman Rubinstein, Analytic methods in the study of zeros of

polynomials...

B. N. Sahney, On the Nörlund summability of Fourier series

Tôru Saitô, Regular elements in an ordered semigroup . .

Lee Meyers Sonneborn, Level sets on spheres...........

Charles Andrew Swanson, Asymptotic estimates for limit point

problems .

Lucien Waelbroeck, On the analytic spectrum of Arens . .

Alvin (Murray) White, Singularities of a harmonic function of three

variables given by its series development .............

Kōichi Yamamoto, Decomposition fields of difference sets ...

Chung-Tao Yang, On the action of $\mathrm{SO}(3)$ on a cohomology manifold... 